\title{
Sternal Retraction and Subclavian Vein Catheter Occlusion during Cardiac Surgery
}

\author{
Masoud Tarbiat, M.D., Ph.D. ', Mohammad Hossein Bakhshaei, M.D., Ph.D.', Amir Derakhshanfar, M.D., Ph.D. ${ }^{2}$, \\ Mahmoud Rezaei, M.D., Ph.D.', Manoochehr Ghorbanpoor, M.D., Ph.D.', Seyed Mohammad Zolhavarieh, M.D., Ph.D'. \\ Departments of 'Anesthesiology and ${ }^{2}$ Surgery, School of Medicine, Clinical Research Development Unit of Farshchian Hospital, School of Medicine, Hamadan \\ University of Medical Sciences, Hamadan, Iran
}

\section{ARTICLE INFO}

Received May 11, 2021

Revised July 13, 2021

Accepted July 16, 2021

Corresponding author

Seyed Mohammad Zolhavarieh

Tel 98-91-2314-7552

Fax 98-511-3852-5307

E-mail Sm.zolhavarieh@umsha.ac.ir

ORCID

https://orcid.org/0000-0003-3401-7145
Background: Subclavian vein (SV) catheterization is a method for the delivery of fluids, drugs, and blood products, venous blood sampling, and central vein pressure monitoring in cardiac surgery. Catheter occlusion is a serious complication of SV catheterization during cardiac surgery, especially after sternal retractor expansion.

Methods: In this observational study, 303 patients who had successful right infraclavicular SV catheterization from September 2019 to April 2020 were enrolled to determine the incidence of catheter occlusion. After catheterization, the lumens of all catheters were checked for the ability to infuse and withdraw blood from the catheter before and after sternal retractor expansion. The patients' characteristics, cannulation approach, on-pump or off-pump technique, occlusion of the catheter and its lumens, and any associated complications were recorded. The data were analyzed using IBM SPSS ver. 22.0 (IBM Corp., Armonk, NY, USA).

Results: Of the 303 patients studied, 205 were male (67.7\%) and 98 were female (32.3\%). Catheter occlusion occurred in 11 patients with on-pump cardiopulmonary bypass (CPB) (227 patients) and 4 patients with off-pump CPB (76 patients) ( $\mathrm{p}=0.863$ ). The incidence of catheter occlusion was $4.95 \%$ (15 of 303 patients) with no cases of simultaneous 3-lumen occlusion in a catheter. The most commonly occluded lumen was the distal lumen (57.92\%). Simultaneous 2-lumen occlusion occurred in 4 patients. Catheter occlusion was found in 3 of 13 malpositioned catheters (23.07\%).

Conclusion: The current study showed that malpositioning of the catheter tip was a risk factor for catheter occlusion and that the distal lumen of a triple-lumen catheter was the most commonly occluded lumen.

Keywords: Complication, Subclavian vein, Catheterization, Catheter obstruction, Central venous catheters

\section{Introduction}

Central venous catheterization is a routine component of modern cardiac surgery. At present, there are 2 main catheterization techniques (ultrasound-guided and anatomic landmark-based), both of which are used by physicians. Central venous catheterization is generally used for both hemodynamic monitoring (central venous pressure [CVP]) and the rapid administration of fluids and medications. Infraclavicular subclavian vein (SV) catheterization may sometimes be used for this purpose in cardiac surgery. As the catheter usually stays in place for more than a week, it causes patient discomfort (especially after internal jugular vein catheterization) [1-3]. In addition to routine internal jugular vein catheterization, SV catheterization is sometimes used at Farshchian Cardiovascular Hospital, Hamadan, Iran. It has some advantages, such as the cosmetic appearance of the neck (lack of a scar from the suture in the neck due to catheter fixation in internal jugular vein cannulation), ease of neck motion, lack of serious neurological damage in high-risk atherosclerotic cardiac patients due to the risk of inadvertent carotid artery puncture during in- 
ternal jugular vein catheterization, a lower risk of infection, and improved nursing care in cardiac surgery. However, SV catheterization has a few well-known complications, such as inadvertent subclavian artery puncture, catheter tip malpositioning, hemothorax, pneumothorax, and catheter occlusion [4-6]. Catheter occlusion refers to the inability to infuse medications and fluids or withdraw fluids or blood from the catheter after sternal retractor expansion during cardiac surgery $[3,6]$. Catheter occlusion is a very important issue in patient management during cardiac surgery. However, few studies in the literature have addressed this issue. This gap in the research prompted us to investigate SV catheter occlusion after sternal retractor expansion during cardiac surgery.

\section{Methods}

The protocol of the present study was evaluated and approved by the Ethics Committee of Hamadan University of Medical Sciences (IR.UMSHA.REC.1398.852).

After obtaining institutional ethical committee approval, this prospective observational study was conducted among 326 patients aged 25-89 years categorized as American Society of Anesthesiologists class II or III who underwent SV catheterization during cardiac surgery from September 2019 to April 2020. Written informed consent was obtained from all patients before the study. The routine evaluation prior to catheterization included a chest X-ray and assessments of prothrombin time (PT), international normalized ratio (INR), partial thromboplastin time (PTT), and platelet count. The exclusion criteria included emergency surgery, left or supraclavicular SV catheterization, internal jugular vein catheterization, concurrent carotid endarterectomy, history of radiotherapy, blood coagulopathy (abnormal PT/PTT/INR or platelet count), hematoma or infection in the region of SV cannulation, a history of catheterization or surgery at the puncture site, and failure of infraclavicular SV catheterization. In all cases, SV catheterization was performed with the patient in the Trendelenburg position with the arms kept by the side after tracheal intubation. The patient was disconnected from mechanical ventilation during needle insertion. After 2 unsuccessful attempts (each skin puncture was defined as an attempt) of right infraclavicular SV catheterization, the patient was excluded from the study. Catheterization was carried out by right-handed physicians using the anatomic landmark-based technique (midpoint [MP] or lateral [LA] blind approach) according to the physician's preference. The modified Selinger technique was used for the MP ap- proach (puncture site: 1 to $2 \mathrm{~cm}$ beneath the MP of the clavicle) or the LA approach (1 to $2 \mathrm{~cm}$ LA to the midclavicular line) after puncturing the SV [7-9]. The length of all triple-lumen catheters was $20 \mathrm{~cm}$ for insertion. All catheters (7F, triple-lumen central catheters) were inserted to a depth of $15 \mathrm{~cm}$ from the skin. The return of venous blood into the syringe attached to the needle confirmed entry into the vessel. Then, all 3 lumens of the catheter were checked for blood aspiration. If blood aspiration was not successful, the catheter was pulled back slowly to the point that allowed free blood aspiration and then fixed at that level. Finally, the wing and the butterfly clamp of the catheter were sutured to the skin in a straight line (Fig. 1). A Cooley sternal retractor was used for expansion of the sternum in all patients. All lumens of the catheter were also rechecked for the ability to infuse and withdraw fluids or blood from the catheter before and after sternal retractor expansion during cardiac surgery. In all cardiac operations, heparin was used and the activated clotting time was checked and kept above 480 seconds in on-pump conditions and $>300$ seconds in off-pump conditions. A portable chest X-ray examination was performed in the intensive care unit immediately after surgery to evaluate potential complications and determine the position of the catheter tip. The variables analyzed in this study were age, sex, weight, height, body mass index (BMI), the cannulation approach, and probable complications such as malposition of the catheter tip, pneumothorax, hemothorax, subclavian arterial puncture, hematoma at the puncture site, and evaluation of the lumens of the catheters for the ability to in-

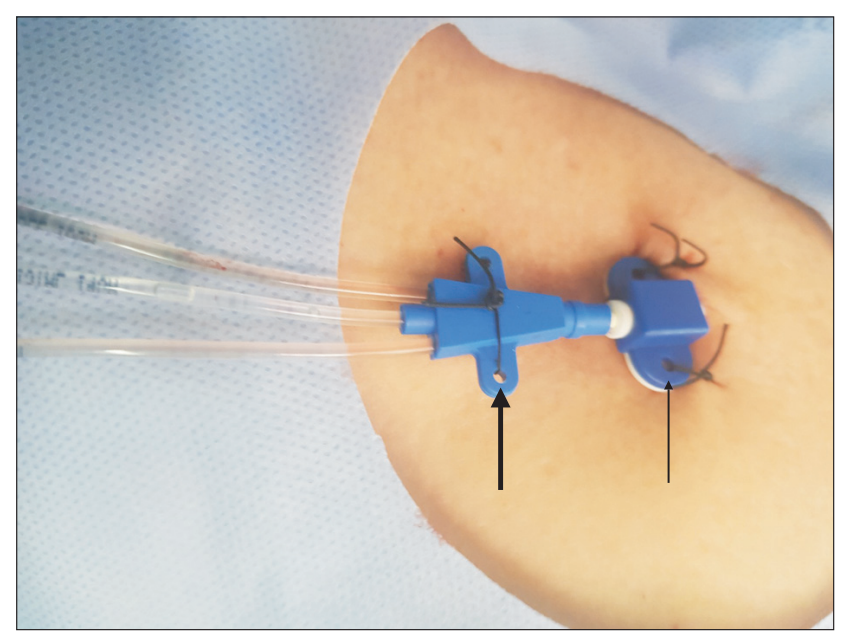

Fig. 1. Fixation of the suture of the wing (thick arrow) and butterfly clamp (narrow arrow) by the one time and conventional (classic) method to the skin in a straight line. A written informed consent for publication of this image was obtained from the patient. 
fuse fluids or blood after sternal retractor expansion.

The Kolmogorov-Smirnov normality test showed the normality of continuous data. The chi-square test was used to evaluate differences between groups in categorical (qualitative) variables. Furthermore, the t-test was carried out to evaluate the differences between mean values of continuous variables. A p-value $<0.05$ was considered to indicate statistical significance. All statistical calculations were performed using IBM SPSS ver. 22.0 (IBM Corp., Armonk, NY, USA).

\section{Results}

Of the 326 cardiac patients who were initially enrolled in this study, 23 were excluded due to failure of right SV catheterization. Failure in 2 attempts occurred in 9 patients (5.2\%) with the MP approach and 14 patients (10.7\%) with the LA approach. Among the 303 included patients, 205 (67.7\%) were men and 98 (32.3\%) were women; their mean age was $61.59 \pm 11.33$ years. The patients' demographic information is shown in Table 1.

Puncture of the subclavian artery occurred in 7 patients: 4 using the MP approach and 3 using the LA approach. In 8 patients, pneumothorax occurred (6 using the MP approach and 2 using the LA approach).

Thirteen of the 303 catheters ( 9 using the MP approach and 4 using the LA approach) placed through the right SV were misplaced. In 8 of these 13 cases, the catheter tip was placed in the right internal jugular vein (6 using the MP approach and 2 using the LA approach). The other catheter tips $(n=5)$ were placed in the left SV (3 using the MP approach and 2 using the LA approach). In 227 of the 303 patients $(74.92 \%)$, cardiac surgery was performed with cardiopulmonary bypass (CPB) (on-pump), while in 76 patients $(25.08 \%)$ it was performed without CPB (offpump). Coronary artery bypass grafting (CABG) was performed in 257 patients (181 on-pump and 76 off-pump CABG). SV catheter occlusion occurred in 15 patients (4.95\%) after sternal retractor expansion during cardiac surgery (11 in on-pump and 4 in off-pump cardiac surgery). There was no significant difference between SV catheter occlusion between on-pump and off-pump cardiac surgery ( $\mathrm{p}=0.863)$. In only 1 patient, SV catheter occlusion occurred during simultaneous CABG and aortic valve replacement surgery. There was also no significant difference between the MP (6.94\%) and LA approaches (5.38\%) in terms of catheter occlusion $(\mathrm{p}=0.581)$ (Table 2). Catheter occlusion occurred in 19 lumens (6.27\%). The most commonly occluded lumen was the distal lumen (57.92\%). Simultaneous 2-lumen occlusion occurred in 4 patients (Table 3), but there were no cases of concurrent 3-lumen occlusion. SV catheter occlusion occurred in 3 of 13 malpositioned catheters (23.07\%). Moreover, in 1 malpositioned catheter (right internal jugular vein), despite an inability to aspirate blood from the distal lumen, there was no inability to infuse medications and fluids. In 1 patient,

Table 1. Patient characteristics

\begin{tabular}{lcccc}
\hline \multicolumn{1}{c}{ Characteristic } & MP group $(\mathrm{n}=173)$ & LA group $(\mathrm{n}=130)$ & Total $(\mathrm{n}=303)$ & $\mathrm{p}$-value \\
\hline Sex & & & & \\
$\quad$ Male & 113 & 92 & 985 & \\
Female & 60 & $61.18 \pm 10.89$ & $61.59 \pm 11.33$ & 0.587 \\
Age $(\mathrm{yr})$ & $61.90 \pm 11.57$ & $70.06 \pm 11.49$ & $70.37 \pm 11.97$ & 0.682 \\
Weight $(\mathrm{kg})$ & $70.61 \pm 12.35$ & $163.64 \pm 8.71$ & $163.57 \pm 10.13$ & 0.911 \\
Height $(\mathrm{cm})$ & $163.51 \pm 11.11$ & $26.15 \pm 3.76$ & $26.67 \pm 8.92$ & 0.587 \\
Body mass index $\left(\mathrm{kg} / \mathrm{m}^{2}\right)$ & $27.07 \pm 12.35$ & & & \\
\hline
\end{tabular}

Values are presented as number or mean \pm standard deviation.

MP, midpoint approach; LA, lateral approach.

Table 2. Complications of catheterization

\begin{tabular}{lrrrr}
\hline \multicolumn{1}{c}{ Complications } & MP group & LA group & Total & p-value \\
\hline Malposition & $9(5.20)$ & $4(3.08)$ & $13(6.27)$ & 0.366 \\
Subclavian artery puncture & $4(2.31)$ & $3(2.30)$ & $7(2.31)$ & 0.998 \\
Pneumothorax & $6(3.47)$ & $2(1.54)$ & $8(2.64)$ & 0.739 \\
Catheter occlusion & $12(6.94)$ & $7(5.38)$ & $19(6.27)$ & 0.581 \\
\hline
\end{tabular}

Values are presented as number (\%).

MP, midpoint approach; LA, lateral approach. 
Table 3. Lumen occlusion

\begin{tabular}{lc}
\hline \multicolumn{1}{c}{ Lumen occlusion } & No. $(\%)$ \\
\hline Distal (14G) & $11(57.92)$ \\
Medial (18G) & $2(10.52)$ \\
Proximal (18G) & $2(10.52)$ \\
Distal and medial & $2(10.52)$ \\
Distal and proximal & $2(10.52)$ \\
Total & $19(100.00)$ \\
\hline
\end{tabular}

proximal lumen occlusion occurred in the intensive care unit, not in the operating room. There was no significant relationship between BMI and lumen occlusion $(p=0.319)$ (Table 4). Furthermore, there were no cases of thoracic duct injury, hematoma at the puncture site, or hemothorax after infraclavicular SV catheterization.

\section{Discussion}

In cardiac surgery, the ability to measure the CVP and infuse medications (especially inotropes) and fluids via a central vein catheter is an essential part of patient care in the operating room and intensive care unit. The SV remains attractive as a site for central venous cannulation due to its greater diameter, easy insertion, higher level of patient acceptance, and lower risk of catheter-associated infection and thrombosis than the femoral or internal jugular vein for some physicians. In addition, the point of insertion is in a wide, flat region of the chest that makes it ideal for use in central venous cannulation. Moreover, as the SV is suspended in the soft tissue underlying the clavicle bone, SV cannulation causes much less vein collapse than internal jugular cannulation. The consequences of vein collapse are back wall puncture, hemorrhage, hematoma, and extravenous placement of the dilator, guide wire or catheter [5,10-12].

Catheter occlusion is a cause of malfunction of the catheter. There are 3 degrees of occlusion. The first is termed partial occlusion (sluggish flow through the catheter and resistance to flushing and aspiration), the second is known as withdrawal occlusion (able to infuse fluids or blood without resistance and unable to withdraw blood) and the third is complete occlusion (unable to infuse or withdraw fluids or blood) $[13,14]$. Catheter occlusion can also be caused by either thrombotic or non-thrombotic events [15]. The non-thrombotic causes of catheter occlusion are kinking (folding of the catheter on itself) or bending of the catheter, fragmentation or fracture of the catheter, pinchoff syndrome (crimping of the catheter between clavicle
Table 4. BMI and lumen occlusion

\begin{tabular}{cccc}
\hline \multicolumn{1}{c}{ Variable } & $\begin{array}{c}\text { Lumen } \\
\text { occlusion }\end{array}$ & $\begin{array}{r}\text { No lumen } \\
\text { occlusion }\end{array}$ & p-value \\
\hline BMI $\left(\mathrm{kg} / \mathrm{m}^{2}\right)$ & & & 0.319 \\
$<19(\mathrm{n}=10)$ & 0 & $10(3.08)$ & \\
$19-25(\mathrm{n}=117)$ & $11(9.4)$ & $106(90.6)$ & \\
$25-30(\mathrm{n}=123)$ & $5(4.1)$ & $118(95.9)$ & \\
$>30(\mathrm{n}=53)$ & $3(5.7)$ & $50(94.3)$ & \\
Total $(\mathrm{n}=303)$ & $19(6.3)$ & $284(93.7)$ & \\
\hline
\end{tabular}

Values are presented as number (\%). $\mathrm{BMI}$, body mass index.

and first rib), a tight suture, a catheter clamp accidentally left closed, and catheter malposition [16-18]. In addition to the above-mentioned causes, catheter occlusion in cardiac surgery can occur through external kinking of the catheter if the surgeon inadvertently places external pressure on the exposed catheter or kinking from the outward and upward pull created by the sternal retractor (similar to the anatomic deformity of the first rib in cases of thoracic outlet syndrome) $[13,19]$.

Other factors could influence catheter occlusion during sternal retraction, such as the type of sternal retractor, obstruction related to low $\mathrm{pH}$ (hydrochloric acid, $0.1 \mathrm{~mol} / \mathrm{L}$ ) or high $\mathrm{pH}$ (sodium bicarbonate, $1.0 \mathrm{~mol} / \mathrm{L}$ ) drugs, the type of fixation of the catheter, and the length and type of the catheter $[17,20]$.

After an extensive literature search, we found no study that focused on catheter occlusion following infraclavicular SV catheterization during cardiac surgery. However, catheter occlusion following SV cannulation was mentioned in a few studies. For example, Sofue et al. [16] stated that it was not possible to inject fluid via the central venous port (left and right subclavian or right internal jugular vein) in 15 of 1,546 oncologic patients (0.97\%). The central venous ports were implanted via the right internal jugular vein $(n=36)$, left SV $(n=1,083)$, or right SV $(n=427)$. Mantia et al. [19] investigated the relationship between sternal retraction and the incidence of permanent loss of pulmonary artery (PA) and CVP waveforms after sternal retractor expansion in 41 cardiac patients in 1988. They described the loss of the PA and CVP waveforms after sternal retractor expansion in $40 \%$ of left SV catheterizations (6 of 15) and $60 \%$ of right SV catheterizations (3 of 5). Thakur et al. [21] also reported 1 case of kinking of the catheter in $30(3.33 \%)$ of elective or emergency surgery patients using the landmark-based infraclavicular SV technique. Kocum et al. [4] mentioned that it was difficult to infuse fluids via a central venous catheter $(7 \mathrm{~F}, 3$-lumen, $20-\mathrm{cm})$ in 14 of 65 cardiac 
patients (21\%) through the MP infraclavicular SV approach. They used a Morse sternal retractor for sternum expansion during cardiac surgery.

There have also been several reports about catheter occlusion due to knotting, bending, or kinking the catheter or its lumens. The catheter must be fixed straight, without any bends, to prevent kinking, especially for soft and flexible catheters $[15,22,23]$. The assessment of catheter occlusion involves inspecting the catheter and administration set for signs of kinked or clamped tubing, catheter tip malposition, loose tubing connections, and tight sutures. Chest $\mathrm{X}$-ray is a valuable tool for assessing pinch-off syndrome and kinking or positioning of the catheter. The management of catheter occlusion involves repositioning the sternal retractor (if possible), resolving the kinking of the catheter, loosening tight stitches, and replacing the catheter $[17,20,24]$. In our study, catheter tip malposition was a risk for catheter occlusion (23.07\%). In addition, as in a previous report, inability to aspirate blood from the lumen occurred in malpositioned catheters [25]. Therefore, negative findings for blood aspiration from any of the lumens must be considered a sign of catheter tip malposition. The incidence of catheter occlusion was $4.95 \%$, and there were no cases of concurrent 3-lumen occlusion in the catheters. The discordant results across studies may be due to differences in the number of patients included in the studies, the point of catheterization, catheter characteristics, the type of sternal retractor, and the method of fixation and securing the catheter. A limitation of this study is that catheter occlusion was evaluated for cases of right SV catheterization, meaning that the study results may not apply to left SV catheterization. Moreover, we believe that further clinical trials and similar studies are needed at various cardiac surgery centers to evaluate SV catheter occlusion in cardiac surgery.

In conclusion, the findings of this study indicated that the distal lumen of the triple catheter $(20 \mathrm{~cm})$ was the most commonly occluded lumen and that none of the catheters simultaneously had 3 occluded lumens. Moreover, malpositioning of the catheter tip was a serious risk factor for catheter occlusion. Ultimately, because all catheter lumens are commonly used during surgery (for rapid administration of drugs, blood products, and fluids), patency of the lumens is critical. Therefore, if physicians consider infraclavicular SV catheterization for cardiac surgery, they must be aware of this serious complication.

\section{Conflict of interest}

No potential conflict of interest relevant to this article was reported.

\section{Acknowledgments}

The authors gratefully acknowledge the Research Vice Chancellor of Hamadan University of Medical Sciences for the material and support for this study and also gratefully thank Dr. Roohi Jamal (Fatemeh) Chakoosari for reviewing and editing this manuscript.

\section{Funding}

The Hamadan University of Medical Sciences supported the study.

\section{ORCID}

Masoud Tarbiat: https://orcid.org/0000-0002-0025-9771

Mohammad Hossein Bakhshaei:

https://orcid.org/0000-0002-3820-2447

Amir Derakhshanfar: https://orcid.org/0000-0002-4096-8740

Mahmoud Rezaei: https://orcid.org/0000-0003-2123-1839

Manoochehr Ghorbanpoor:

https://orcid.org/0000-0002-0645-3890

Seyed Mohammad Zolhavarieh:

https://orcid.org/0000-0003-3401-7145

\section{References}

1. Leal MLM, Loyola ABAT, Hueb AC, et al. Fixation of the short-term central venous catheter: a comparison of two techniques. Acta Cir Bras 2017;32:680-90.

2. Uemura K, Inoue S, Kawaguchi M. The unnecessary application of central venous catheterization in surgical patients. Braz J Anesthesiol 2018;68:336-43.

3. Tarbiat M, Davoudi M, Salimbahrami SA. Influence of arm position during infraclavicular subclavian vein catheterization in coronary artery bypass graft surgery. J Cardiovasc Thorac Res 2018;10:192-6.

4. Kocum A, Sener M, Caliskan E, Bozdogan N, Atalay H, Aribogan A. An alternative central venous route for cardiac surgery: supraclavicular subclavian vein catheterization. J Cardiothorac Vasc Anesth 2011; 25:1018-23.

5. Tarbiat M, Farhanchi A, Davoudi M, Farhadian M. Supraclavicular versus infraclavicular subclavian vein catheterization in coronary artery bypass graft surgery. Res Cardiovasc Med 2018;7:5-9.

6. Czarnik T, Gawda R, Perkowski T, Weron R. Supraclavicular ap- 
proach is an easy and safe method of subclavian vein catheterization even in mechanically ventilated patients: analysis of 370 attempts. Anesthesiology 2009;111:334-9.

7. Tarbiat M, Salimbahrami SA, Khorshidi HR. Influence of cannulation point on infraclavicular subclavian vein catheterization: a clinical trial. Anesth Pain Med 2019;9:e92724.

8. Tan BK, Hong SW, Huang MH, Lee ST. Anatomic basis of safe percutaneous subclavian venous catheterization. J Trauma 2000;48:826.

9. Heffner AC, Androes MP. Placement of subclavian venous catheters [Internet]. Waltham (MA): UpToDate; 2019 [cited 2021 May 10]. Available from: https://www.uptodate.com/contents/placement-ofsubclavian-venous-catheters.

10. Aziz N, Khan A, Iqbal J. Subclavian vein catheterization: supraclavicular versus infraclavicular approach. J Med Sci 2013;21:187-9.

11. Tarbiat M, Manafi B, Davoudi M, Totonchi Z. Comparison of the complications between left side and right side subclavian vein catheter placement in patients undergoing coronary artery bypass graft surgery. J Cardiovasc Thorac Res 2014;6:147-51.

12. Bannon MP, Heller SF, Rivera M. Anatomic considerations for central venous cannulation. Risk Manag Healthc Policy 2011;4:27-39.

13. Cummings-Winfield C, Mushani-Kanji T. Restoring patency to central venous access devices. Clin J Oncol Nurs 2008;12:925-34

14. Baskin JL, Reiss U, Wilimas JA, et al. Thrombolytic therapy for central venous catheter occlusion. Haematologica 2012;97:641-50.

15. Yoon KB, Kim WO, Cha JH, Lee KY. Malfunction due to kinking and banding of a double lumen central venous catheter: a case report. Korean J Crit Care Medicine 2006;21:131-4.

16. Sofue K, Arai Y, Takeuchi Y, Sugimura K. Flow confirmation study for central venous port in oncologic outpatient undergoing chemotherapy: evaluation of suspected system-related mechanical complications. Eur J Radiol 2013;82:e691-6.

17. Baskin JL, Pui CH, Reiss U, et al. Management of occlusion and thrombosis associated with long-term indwelling central venous catheters. Lancet 2009;374:159-69.

18. Keum DY, Kim JB, Chae MC. Safety of a totally implantable central venous port system with percutaneous subclavian vein access. Korean J Thorac Cardiovasc Surg 2013;46:202-7.

19. Mantia AM, Robinson JN, Lolley DM, Nieman HL, Berkebile PE, Stullken EH Jr. Sternal retraction and pulmonary artery catheter compromise. J Cardiothorac Anesth 1988;2:430-9.

20. Hill J, Broadhurst D, Miller K, et al. Occlusion management guideline for central venous access devices (CVADs). Vasc Access 2013; 7(Suppl 1):1-36.

21. Thakur A, Kaur K, Lamba A, et al. Comparative evaluation of subclavian vein catheterisation using supraclavicular versus infraclavicular approach. Indian J Anaesth 2014;58:160-4.

22. Raut MS, Maheshwari A. Undiagnosed kinking of advanced venous access. Glob Anesth Perioper Med 2016;2:178.

23. Cho HB, Kim SH, Yoo JH, et al. Malfunction of a central venous multilumen access catheter caused by kinking: a case report. Medicine (Baltimore) 2018;97:e11622.

24. Bhutta ST, Culp WC. Evaluation and management of central venous access complications. Tech Vasc Interv Radiol 2011;14:217-24.

25. Hayaran N, Goyal N, Joy S, Jain A. Coiling of central venous catheter: a rare and preventable complication. Anesth Essays Res 2017;11: 773-5. 